\title{
Effects of electromagnetic forcing on self-sustained jet oscillations
}

\author{
R. Kalter, ${ }^{1, a)}$ M. J. Tummers, ${ }^{2}$ S. Kenjereš, ${ }^{1}$ B. W. Righolt, ${ }^{1}$ and C. R. Kleijn ${ }^{1}$ \\ ${ }^{1}$ Department of Chemical Engineering, Delft University of Technology, Julianalaan 136, \\ 2628 BL Delft, The Netherlands \\ ${ }^{2}$ Laboratory for Aero and Hydrodynamics, Delft University of Technology, \\ Leeghwaterstraat 21, 2628 CA Delft, The Netherlands
}

(Received 27 February 2014; accepted 20 May 2014; published online 6 June 2014)

\begin{abstract}
The influence of electromagnetic forcing on self-sustained oscillations of a jet issuing from a submerged nozzle into a thin vertical cavity (width $W$ much larger than thickness $T$ ) has been studied using particle image velocimetry. A permanent Lorentz force is produced by applying an electrical current across the width of the cavity in conjunction with a magnetic field from three permanent magnets across its thickness. As a working fluid a saline solution is used. The magnetic field is in the north-southnorth configuration, such that the Lorentz force can be applied in an up-down-up configuration or in a down-up-down configuration by switching the direction of the electrical current. A critical Stuart number $\mathrm{N}_{c}$ was found. For $\mathrm{N}<\mathrm{N}_{c}$, the jet oscillates with a constant Strouhal number St, independent of the Reynolds number Re. For $\mathrm{N}>\mathrm{N}_{c}$ and an oscillation enhancing up-down-up configuration of the Lorentz force, St grows with $\mathrm{N}$ as $\mathrm{St} \propto \sqrt{\mathrm{N}}$. In contrast, for $\mathrm{N}>\mathrm{N}_{c}$ and an oscillation suppressing down-up-down configuration of the Lorentz force, all jet oscillations are suppressed. (c) 2014 AIP Publishing LLC. [http://dx.doi.org/10.1063/1.4880897]
\end{abstract}

\section{INTRODUCTION}

Self-sustained flow oscillations are known to occur in confined geometries, when shear layers are bounded by recirculation zones. ${ }^{1,2}$ Flows in which self-sustained oscillations occur are (amongst others): jets issuing into a thin cavity, ${ }^{3-9}$ the flow over backward facing steps, ${ }^{10,11}$ and flows over cavities. ${ }^{12-15}$ The self-sustained oscillations have a much lower frequency than those produced by shear layer instabilities.

In the present work we focus on a single jet issuing from a submerged nozzle into a thin cavity. Honeyands and Herbertson ${ }^{4}$ investigated this flow problem earlier and they reported a selfsustained oscillating behavior of the jet. Their results show that the interactions of the recirculation zones alongside the jet with the jet itself are of large importance for the mechanism leading to the self-sustained oscillation. An imbalance was found between the pressure force in the center of the recirculation zone and the inertial force in the jet. These two forces fluctuate with the same frequency as the frequency of the jet oscillation, but a phase difference with a specific delay time exists between the maxima in the pressure force and the inertial force. This delay time is essential in the mechanism behind the self repeating behavior of the jet oscillation. Influencing the formation of the recirculation zones changes the interaction between the recirculation zones and the jet, and hence the jet oscillation can be altered. To achieve this influence on the jet oscillation, we apply an electromagnetic forcing.

Electromagnetic forcing of flows can be introduced by applying an imposed electrical current in conjunction with a magnetic field. The use of this principle was reported in several publications, for instance to delay flow separations, ${ }^{16,17}$ to increase heat transfer, ${ }^{18,19}$ and to increase laminar

\footnotetext{
a)Electronic mail: r.kalter@tudelft.nl
} 
mixing. ${ }^{20-22}$ Electromagnetic actuators are used for the control of turbulent channel flow, ${ }^{23,24}$ for the suppression of vortex shedding behind a cylinder, ${ }^{25,26}$ and to prevent flow separation past an airfoil..$^{27-29}$

The effect of electromagnetic forcing on self-sustained oscillations of a confined jet issuing into a thin cavity is investigated experimentally using particle image velocimetry (PIV). A permanent Lorentz force is imposed on the fluid by an electrical current across the width of the cavity in conjunction with a permanent magnetic field perpendicular to the electrical current. A saline solution is used as a working fluid to increase the electrical conductivity as compared to tap water. The electromagnetic forcing at the position of the recirculation zones alongside the jet is such that it can be in the same or in the opposite direction as the momentum of the liquid flowing in the recirculation zones. This leads to an increase or a decrease in the growth of the recirculation zones and hence to an enhancement or suppression of the jet oscillation.

The objective of the present research is to quantify the influence of the Lorentz force on selfsustained jet oscillations. We look for the presence of a critical value of the Stuart number, above which the flow dynamics are completely dominated by the Lorentz force.

\section{PHYSICAL MODELING}

\section{A. Experimental setup}

The experiment is conducted in a vertical rectangular glass cavity, schematically shown in Figure 1. The inner dimensions of the cavity are height $H \times$ width $W \times$ thickness $T=0.8 \times 0.3$ $\times 0.035 \mathrm{~m}^{3}$ and it is filled to a height of $h=0.7 \mathrm{~m}$. The working fluid is a solution of $10 \mathrm{wt} . \%$ $\mathrm{Na}_{2} \mathrm{SO}_{4}$ in water. The density of the working fluid is $\rho=1.10 \times 10^{3} \mathrm{~kg} / \mathrm{m}^{3}$, the kinematic viscosity is $v=1.27 \times 10^{-6} \mathrm{~m}^{2} / \mathrm{s}$, and the electrical conductivity is $\sigma=7.1 \mathrm{~S} / \mathrm{m}$ (Wolf et al. ${ }^{30}$ ). The fluid is injected downwardly into the cavity through a vertical, submerged nozzle of $0.4 \mathrm{~m}$ length. The inner dimensions of the nozzle are $d \times d=0.01 \times 0.01 \mathrm{~m}^{2}$ and the outer dimensions are 0.02 $\times 0.02 \mathrm{~m}^{2}$. The bulk velocity in the nozzle is $v_{i n}$. The end of the nozzle is positioned at a depth $d_{n}=0.1 \mathrm{~m}$ below the equilibrium free surface level.

Liquid flows out of the cavity through two $0.04 \times 0.035 \mathrm{~m}^{2}$ rectangular holes in the bottom of the cavity into an external flow circuit. The holes are placed off center, such that a light sheet for PIV measurements can enter the measurement volume through the bottom plate. The liquid is recirculated back to the nozzle by a pump to maintain a constant volume of liquid in the cavity. The liquid is kept at a constant temperature of $\mathrm{T}=20^{\circ} \mathrm{C} \pm 0.5^{\circ} \mathrm{C}$ by means of an immersion cooler in the external circuit to guarantee a constant viscosity of the liquid.

A Cartesian coordinate system is defined with its origin located at the equilibrium position of the free liquid surface in the center of the cavity, see Figure 1. The $x$-axis is parallel to the front wall of the cavity, and the $y$-axis is in the upward vertical direction. The $x$ - and $y$-component of the velocity are denoted by $u_{x}$ and $u_{y}$, respectively.

\section{B. Electromagnetic forcing}

Rolled up platinum electrode wires with a length of $0.8 \mathrm{~m}$ and a diameter of $1 \mathrm{~mm}$ are placed in compartments next to the cavity. The potential difference $\Delta \phi=\phi_{1}-\phi_{2}$ between the electrodes can be set to a maximum of $50 \mathrm{~V}$. This potential difference results in a maximum electrical current of $I=4.0$ A between the electrodes. The electrodes are physically separated from the cavity by a porous wall, such that electrical contact is made, but bubbles due to electrolysis are prevented from entering the cavity. The area of the porous wall in the side wall of the cavity is $0.03 \times 0.03 \mathrm{~m}^{2}$. With an electrical current of $I=4.0 \mathrm{~A}$, the mean current density at the position of the porous walls is $|\mathbf{j}|=4.4 \times 10^{3} \mathrm{~A} / \mathrm{m}^{2}$.

Three permanent $\mathrm{NdFeB}$ magnets, each with dimensions $0.04 \times 0.04 \times 0.084 \mathrm{~m}^{3}$, are placed against the back wall of the cavity. The magnets are placed in a north-south-north configuration, such that the resulting Lorentz force is either up-down-up or down-up-down, depending on the direction of the electrical current. The horizontal distance between the centers of the magnets is $D=0.10 \mathrm{~m}$. 


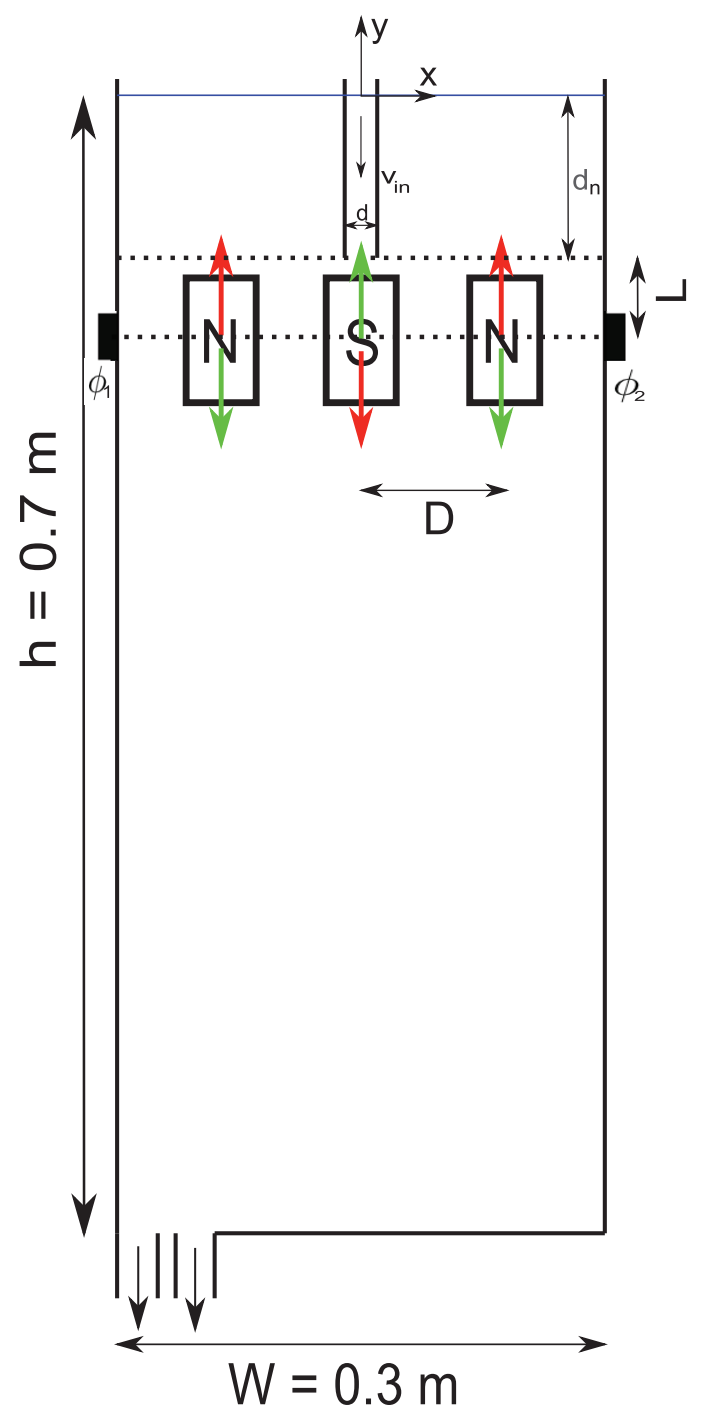

FIG. 1. Experimental flow configuration. The inner dimensions of the cavity are $H \times W \times T=0.8 \times 0.3 \times 0.035 \mathrm{~m}^{3}$. A $10 \% \mathrm{Na}_{2} \mathrm{SO}_{4}$ in water solution flows into the cavity through a nozzle with inner dimensions $d \times d=0.01 \times 0.01 \mathrm{~m}^{2}$ and outer dimensions of $0.02 \times 0.02 \mathrm{~m}^{2}$. The nozzle is submerged to a depth $d_{n}=0.1 \mathrm{~m}$ below the free surface. The cavity is filled up to $h=0.7 \mathrm{~m}$ and the outflow is through two holes of $0.04 \times 0.035 \mathrm{~m}^{2}$ in the bottom of the cavity. Magnets are placed behind the cavity, and the vertical distance between the nozzle and the center of the magnets is denoted by $L$. The horizontal distance between the centers of the magnets is denoted by $D$.

The magnetic field strength in the $z$-direction is measured at $y=-d_{n}-L$, using a Hall-flux sensor and the result is plotted for three values of $z$ in Figure 2. The center of the magnets is located at the same $y$-position as the center of the porous walls. The vertical distance between the nozzle and the center of the magnets is denoted as $L$, as indicated in Figure 1.

\section{PIV system}

A Quantronix Darwin DUO 527-80-M laser and light sheet optics are used to produce a laser light sheet that enters the cavity through the bottom wall. The laser produces $27 \mathrm{~mJ}$ pulses at a wavelength of $527 \mathrm{~nm}$ and a repetition rate of $1000 \mathrm{~Hz}$. Neutrally buoyant silver coated hollow glass spheres with a nominal diameter of $25 \mu \mathrm{m}$ are used as seeding particles. The field of view has size $0.3 \times 0.3 \mathrm{~m}^{2}$ and shows the full width of the cavity $(-0.15 \mathrm{~m}<x<0.15 \mathrm{~m})$ and the region just 


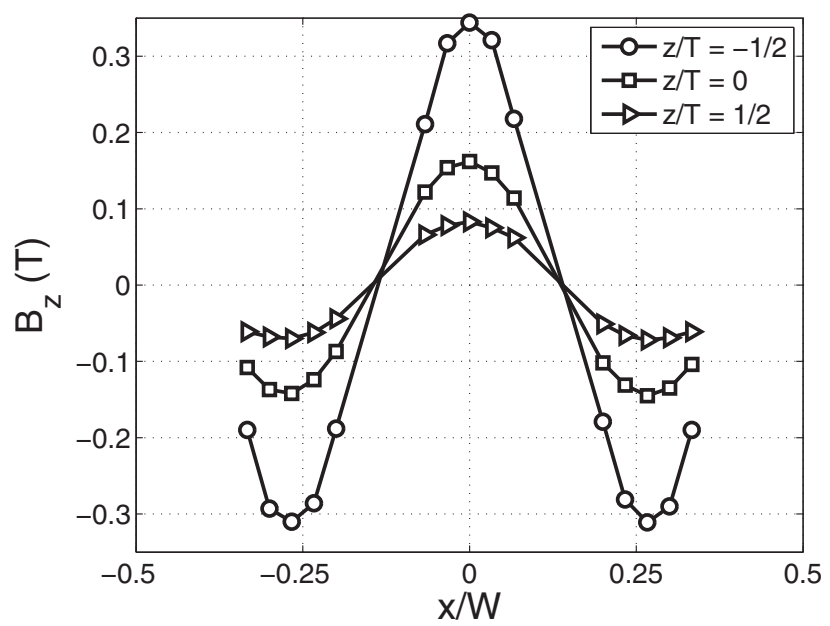

FIG. 2. Measured $z$-component of the magnetic field strength at $y=-d_{n}-L$ for different positions in the $x$ and $z$-directions.

below the nozzle $\left(-d_{n}-0.3 \mathrm{~m}<y<-d_{n}\right)$. The light sheet is located in the $z=0$ plane between the two broad walls of the cavity and the light sheet thickness in the measurement region is less than $1 \mathrm{~mm}$. Particle images are recorded with an Imager HS pro 4M camera with a Nikon Nikkor $55 \mathrm{~mm}$ lens with $f^{\#}=16$. The camera is equipped with a $2016 \times 2016$ pixel CMOS-chip with a pixel size of $11 \times 11 \mu \mathrm{m}^{2}$. Velocity vectors are calculated based on cross correlation of interrogation areas of $32 \times 32$ pixels with $75 \%$ overlap using Davis 8.1 PIV software.

\section{Proper orthogonal decomposition}

Due to the coherent nature of the investigated flows a proper orthogonal decomposition $\left(\mathrm{POD},{ }^{31,32}\right)$ can be applied to find dominant temporal and spatial energy modes in the flow. In the proper orthogonal decomposition, a time series of velocity fields is decomposed as

$$
\mathbf{u}(\mathbf{x}, t)=\sum_{n=0}^{N} a_{n}(t) \phi_{n}(\mathbf{x}),
$$

where the velocity field is expanded into a finite series of orthogonal spatial basis functions $\phi_{n}(\mathbf{x})$ and mutually uncorrelated time coefficients $a_{n}(t)$. Here $n$ denotes the mode number and $\mathrm{N}$ is the number of snapshots from the PIV. The mode $n=0$ is the mean velocity field. The lowest $n>0$ modes are related to the large scale, low frequency fluctuations in the flow. The higher modes are related to the turbulent fluctuations. An overview of the proper orthogonal decomposition is provided in Refs. 31-33.

\section{RELEVANT DIMENSIONLESS NUMBERS}

The dimensionless momentum equation for the incompressible flow in the cavity reads

$$
\frac{\partial \mathbf{u}}{\partial t}+(\mathbf{u} \cdot \nabla) \mathbf{u}=-\nabla p+\frac{1}{\operatorname{Re}} \nabla^{2} \mathbf{u}+\mathrm{N}(\mathbf{j} \times \mathbf{B}),
$$

where $\mathbf{u}$ is the dimensionless fluid velocity, $p$ is the dimensionless pressure, $\mathbf{j}$ is the dimensionless electrical current density, and $\mathbf{B}$ is the dimensionless magnetic field strength. The Lorentz force acting on the flow is given by $\mathbf{F}_{L}=\mathbf{j} \times \mathbf{B}$. For low conducting fluids (e.g., salt water), induced currents $\sigma(\mathbf{u} \times \mathbf{B})$ due to a magnetic field are small and the current density $\mathbf{j}=\sigma(\mathbf{E}+\mathbf{u} \times \mathbf{B}) \approx \sigma \mathbf{E}$ is independent of the local flow velocity. Then the Lorentz force is

$$
\mathbf{F}_{L}=\mathbf{j} \times \mathbf{B} \approx \sigma(\mathbf{E} \times \mathbf{B}) .
$$


Two dimensionless numbers appear in Eq. (2), the Reynolds number:

$$
\operatorname{Re}=\frac{v_{i n} d}{v}
$$

and the Stuart number:

$$
\mathrm{N}=\frac{\sigma E_{r e f} B_{r e f} d}{\rho v_{i n}^{2}} .
$$

The quantities used to determine the values of $\mathrm{N}$ and $\mathrm{Re}$ are the maximum measured magnetic field strength $B_{r e f}=0.15 \mathrm{~T}$ in the $z$-direction at $\left(x / W=0, y=-d_{n}-L, z / T=0\right)$, the nozzle hydraulic diameter $d=0.01 \mathrm{~m}$, the liquid density $\rho=1.10 \times 10^{3} \mathrm{~kg} / \mathrm{m}^{3}$, and the liquid viscosity $v=1.27 \times 10^{-6} \mathrm{~m}^{2} / \mathrm{s}$. The two varied parameters in $\mathrm{N}$ and Re are the current density at the position of the porous walls, $\sigma E_{r e f}=\left|\mathbf{j}_{\text {ref }}\right|$, and the inlet velocity, $v_{i n}$. When $v_{i n}=0 \mathrm{~m} / \mathrm{s}$, the characteristic velocity in the Stuart number is taken to be the maximum measured time-averaged velocity in the vertical direction induced by the Lorentz force above the middle magnet, $v_{\max }$.

A third relevant dimensionless parameter in this study is the Strouhal number, defined as

$$
\mathrm{St}=\frac{f W}{v_{\text {in }}},
$$

which can be interpreted as a dimensionless frequency. The frequency $f$ is made dimensionless using the inlet velocity $v_{i n}$ and the width of the cavity $W$, in accordance with Lawson and Davidson. ${ }^{6}$ As before, in case $v_{i n}=0 \mathrm{~m} / \mathrm{s}$ the characteristic velocity is taken to be $v_{\max }$.

\section{JET OSCILLATIONS WITHOUT LORENTZ FORCING $(\mathrm{N}=0)$}

PIV measurements with a duration of $5 \mathrm{~min}$ and with a sampling rate of $1 \mathrm{fps}$ have been performed in flows with $\Delta \phi=0$, i.e., without an applied Lorentz force. Figure 3 shows four PIV snapshots of the flow for $\operatorname{Re}=7.1 \times 10^{3}$, illustrating one period of a jet oscillation. The oscillation period is about $30 \mathrm{~s}$.
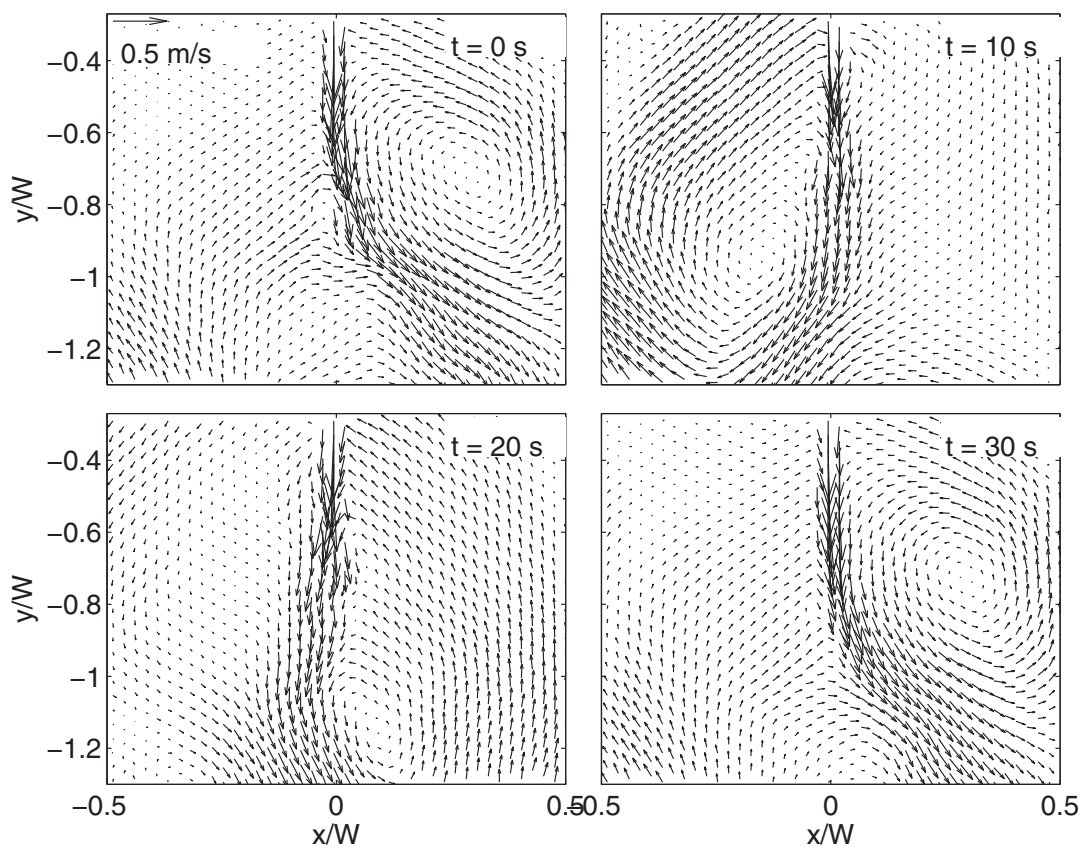

FIG. 3. Jet oscillation at $\mathrm{N}=0, \mathrm{Re}=7.1 \times 10^{3}$. Going from left top to right bottom, the time between the first and the last image is $30 \mathrm{~s}$. The height and width of the images is $0.3 \mathrm{~m}$. 


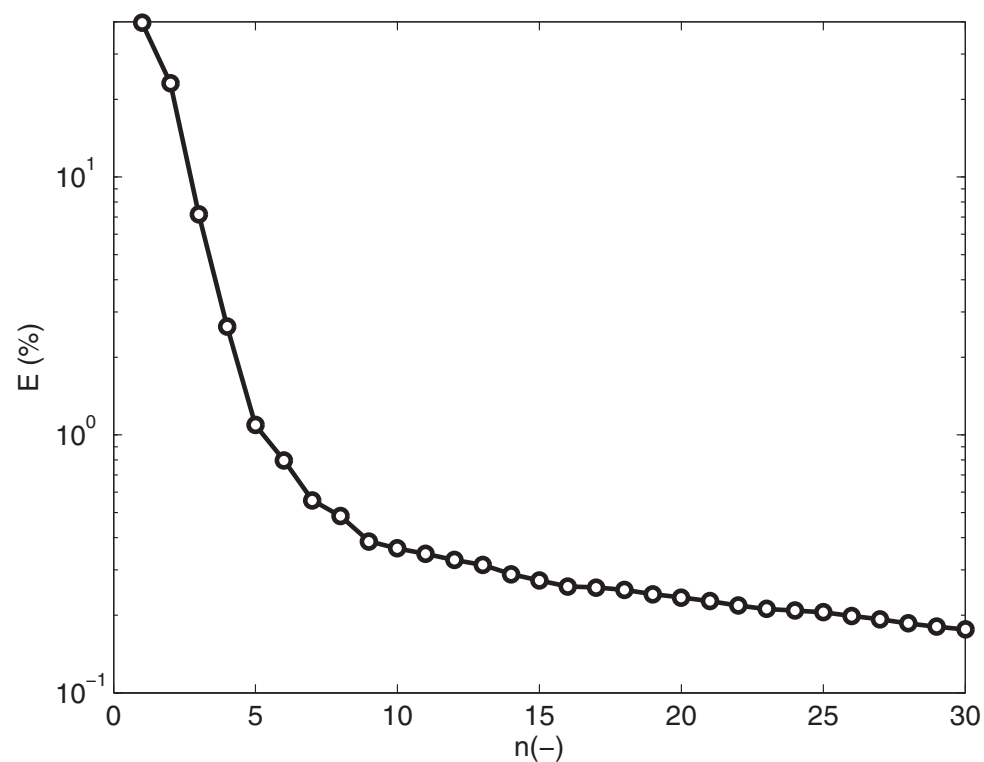

FIG. 4. Energy contained in POD modes 1-30 shown as a percentage of the total kinetic energy for the flow with $\mathrm{Re}=$ $7.1 \times 10^{3}$.

A proper orthogonal decomposition is performed on the PIV snapshots to calculate the first temporal mode $a_{1}(t)$ for different values of the Reynolds number. The temporal mode $a_{1}(t)$ is connected to the most energetic fluctuations in the flow: the large scale jet oscillation. ${ }^{34}$ Figure 4 shows a distribution of the fraction of kinetic energy present in the temporal modes $a_{n}(t)$ of the POD. It is seen that the fraction of energy contained in the first mode $a_{1}(t)$ is $39 \%$. Figure 5 (left) shows a time trace of $a_{1}(t)$ for the flow with $\mathrm{Re}=7.1 \times 10^{3}$ and Figure 5 (right) shows the corresponding power spectrum. A dominant frequency is found at $f=0.033 \mathrm{~Hz}$, in accordance with the oscillation period of $30 \mathrm{~s}$. The Strouhal number is determined with this dominant frequency. The same procedure is applied for measurement results for Reynolds numbers between $\operatorname{Re}=1.5 \times 10^{3}$ and $\operatorname{Re}=3.0 \times$ $10^{4}$. Figure 6 shows that the Strouhal number is independent of the Reynolds number, which is in accordance with results presented in literature..$^{5,6,8,35}$
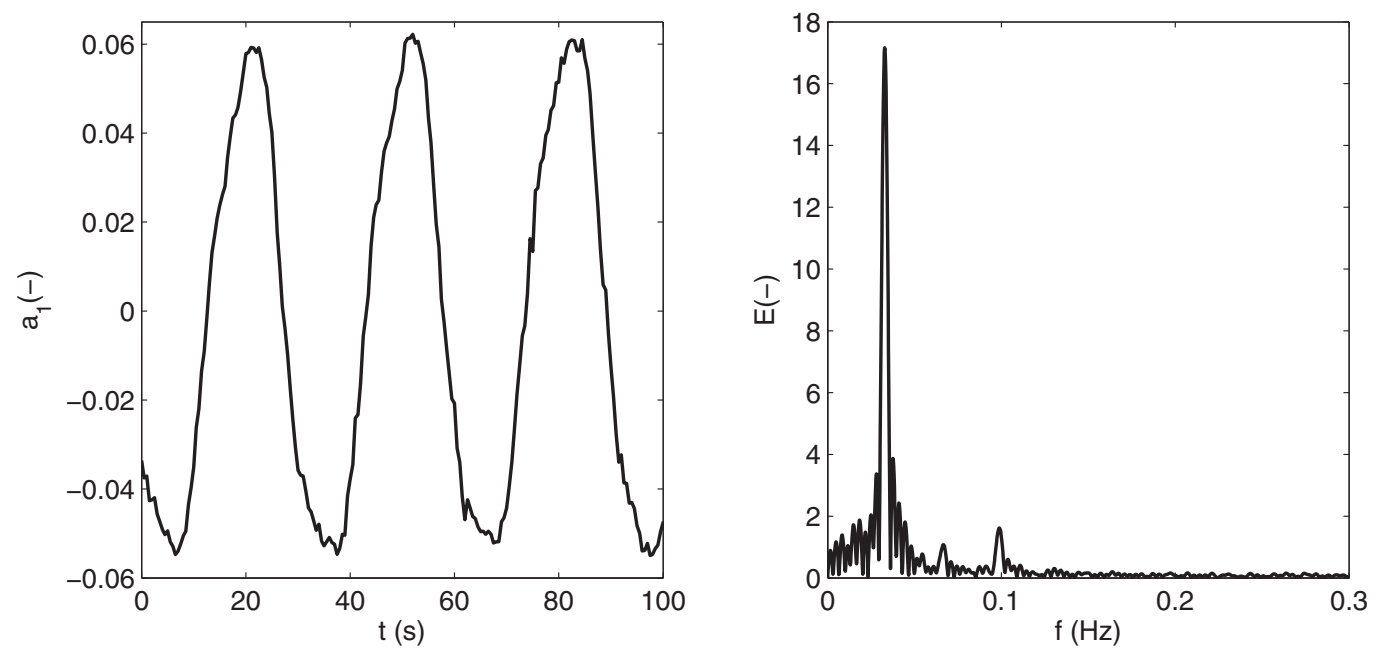

FIG. 5. Left: Time trace of the first temporal mode $a_{1}(t)$ of the POD for Re $=7.1 \times 10^{3}$. Right: Power spectrum of the time trace of $a_{1}(t)$, a clear peak is found at $f=0.033 \mathrm{~Hz}$, with second harmonics at $f=0.067 \mathrm{~Hz}$ and $f=0.099 \mathrm{~Hz}$. 


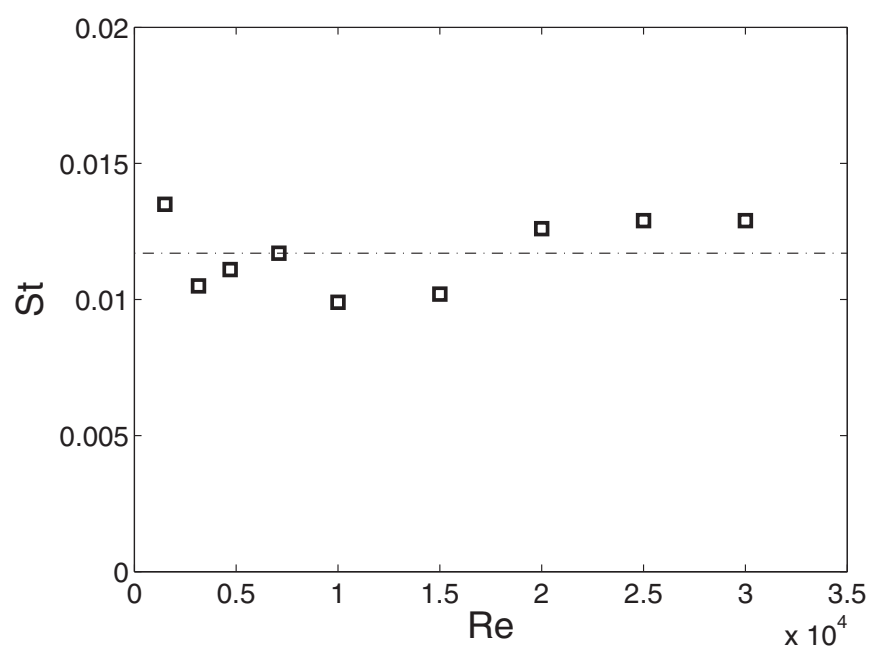

FIG. 6. Strouhal number as a function of Reynolds number for flows with a confined jet without an applied Lorentz force.

\section{PURE LORENTZ FORCING OF THE FLOW $(R e=0)$}

PIV measurements of the flow due to the Lorentz force only (i.e., in absence of jet inflow, $v_{i n}=0$ ) have been performed at a recording rate of one image pair per second and with a duration of 5 min. Figure 7 shows the mean velocity field for an electrical current of $\mathrm{I}=4.0 \mathrm{~A}$ for the up-down-up forcing configuration (left) as well as for the down-up-down forcing configuration (right). It is seen that the flow patterns are reversed due to the reversed electromagnetic forcing. In Figure 7 (right), the free surface is present at $\mathrm{y} / \mathrm{W}=0$, which leads to a confinement of the recirculation zones, while in Figure 7 (left) this is not the case. The magnet positions are indicated by the rectangles. In the remainder of this section we focus on the flow in the up-down-up forcing configuration.

The Lorentz force at the center magnet position is in the downward direction, causing a relatively large flow velocity in the downward direction at $x / W=0$. The Lorentz force at the position of the left and right magnets is in the upward direction, causing an upward directed flow. The combination of the three magnets results in two counter rotating recirculation zones on the left and on the right of the center magnet. The maximum velocity in the flow field, $v_{\max }=0.08 \mathrm{~m} / \mathrm{s}$, is found at $x / W=0$ and $y / W=-0.6$, so below the center of the middle magnet. The centers of the two counter rotating recirculation zones are also located below the position of the center of the middle magnet, at $y / W=-0.4$ and $x / W= \pm 0.2$. Above the magnets on the left and on the right two secondary recirculation zones emerge.
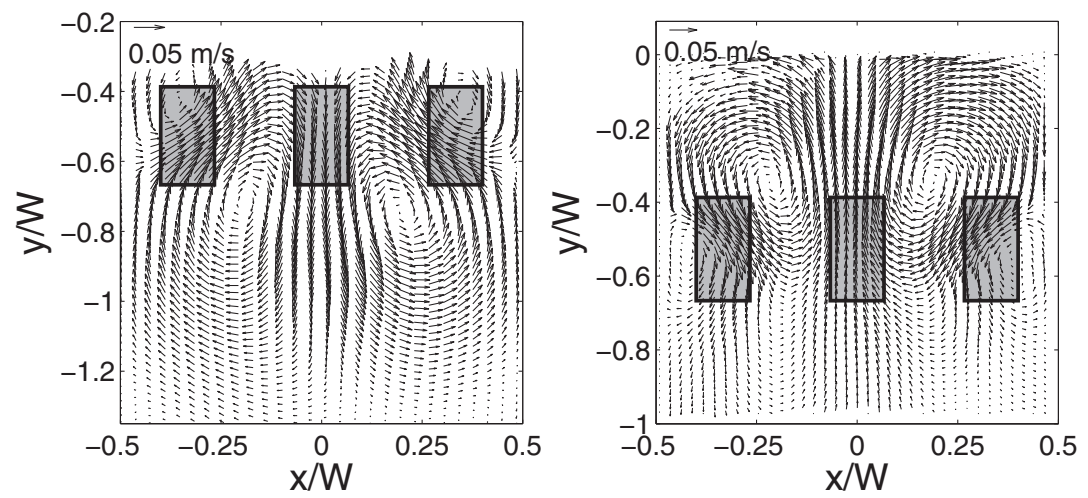

FIG. 7. Mean velocity field for $I=4.0 \mathrm{~A}$ in combination with the magnetic field. Left: up-down-up configuration. Right: down-up-down configuration. The gray rectangles indicate the magnet positions. 


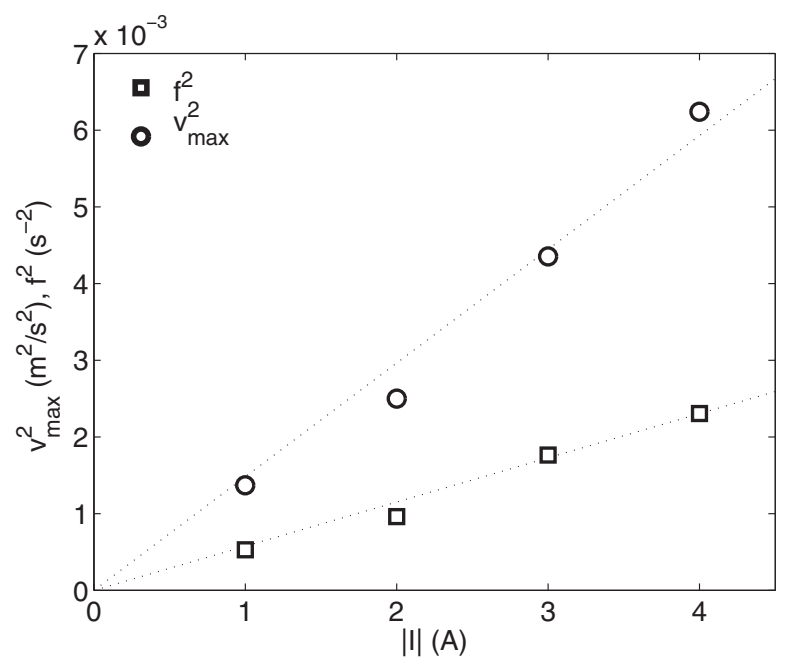

FIG. 8. ( $\square$ ) Square of the dominant frequency in the flow as a function of the electrical current in purely electromagnetically forced flow $\left(v_{i n}=0\right.$ ). (o) Square of the maximum of the mean velocity in the flow as a function of the electrical current in the purely electromagnetically forced flow $\left(v_{i n}=0\right)$.

Figueroa et al. ${ }^{36}$ reported results for flows of saline solutions in shallow layers due to a Lorentz force produced by the combination of a single magnet and an electrical field. They report that the centers of the recirculation zones and the location of the maximum velocity $v_{\max }$ are located downstream of the center of the magnet, which is in agreement with the results presented in Figure 7 (left).

Duran-Matute et al. ${ }^{37}$ reported results for stationary flows of saline solutions due to a Lorentz force produced by three magnets in the south-north-south configuration in combination with an electrical field. Streamwise elongated vortices, similar to the ones observed in Figure 7, were reported. They also found the small recirculation zones above the left and right magnets, shown in the top right and top left corner of Figure 7 (left).

Figure 8 shows $v_{\max }^{2}$ as a function of the applied electrical current. It is observed that $v_{\max } \propto \sqrt{I} \propto \sqrt{\sigma E}$, which is to be expected when the Lorentz force is balanced by the inertial forces: ${ }^{37} v_{\max }=\sqrt{\sigma E B l / \rho}$. For the calculation of $v_{\max }$, the distance between the centers of the magnets $(D=0.1 \mathrm{~m})$ is used as the typical length scale $l$. It is seen that the calculated velocity over estimates the measured velocity by a factor of about 3 , so $v_{\max }=\alpha \sqrt{\sigma E B l / \rho}$, with $\alpha \approx 0.33$. The over estimation of the calculated velocity is expected due to non stationary flow behavior and from an over estimation of the electrical current density $\sigma E$ at the position of the center magnet.

The flow in the cavity induced by the Lorentz forces is non-stationary. The eddies on the left and the right interact with each other, leading to an oscillating behavior of the downward moving flow emerging at the position of the center magnet. The oscillations can exist due to the confined space in the $x$ - and $z$-directions. A proper orthogonal decomposition is performed on the snapshots from the PIV measurement with the up-down-up forcing configuration. From the first temporal mode, $a_{1}(t)$, the dominant frequency of the most energetic fluctuating structures in the flow is determined. Figure 8 shows the square of the frequency as a function of the applied current. The results indicate that the frequency $f$ is proportional to the square root of the electrical current $I$ which means $\operatorname{St} \propto \sqrt{\mathrm{N}}$.

Table I summarizes measured maximum velocities and frequencies, depending on the forced electrical current. In analogy with the self-sustained jet oscillations discussed in Sec. IV, it is expected that $f \propto v_{\max }$, and since $v_{\max } \propto \sqrt{I}$ it follows that $f \propto \sqrt{I}$. This would lead to a constant Stuart number $\mathrm{N}$ and to a constant Strouhal number St, as can indeed be seen in Table I. The constant of proportionality between the square root of the Stuart number and the Strouhal number is approximately 0.1 :

$$
\mathrm{St} \approx 0.1 \sqrt{\mathrm{N}}
$$


TABLE I. Maximum of the mean velocity $v_{\max }$, dominant frequency $f$, Strouhal number St, and the Stuart number $\mathrm{N}$ for various values of the electrical current $I$.

\begin{tabular}{lcccc}
\hline \hline$|I|(\mathrm{A})$ & $v_{\max }(\mathrm{m} / \mathrm{s})$ & $f(\mathrm{~Hz})$ & $\mathrm{N}$ & $\mathrm{St}$ \\
\hline 1.0 & 0.037 & 0.023 & 3.48 & 0.186 \\
2.0 & 0.050 & 0.031 & 3.81 & 0.182 \\
3.0 & 0.066 & 0.042 & 3.28 & 0.190 \\
4.0 & 0.079 & 0.048 & 3.06 & 0.182 \\
\hline \hline
\end{tabular}

Contrary to the present experiment, no flow oscillations were reported by Figueroa et al..$^{36}$ and by Duran-Matute et $a l .{ }^{37}$ This can be expected since in their experiments, there was no confinement of the vortices in the $x$ - or $y$-directions.

\section{MIXED FORCING (N $>0, R e>0)$ : UP-DOWN-UP}

In Secs. IV and V we discussed the two limiting cases of zero jet flow $(\operatorname{Re}=0)$ and zero Lorentz force $(\mathrm{N}=0)$, respectively. This section presents results from measurements with combined jet flow $(\operatorname{Re}>0)$ and flow induced by the Lorentz force $(\mathrm{N}>0)$. Figure 9 shows $\mathrm{St}$ as a function of $\mathrm{N}$ being independent of the dimensionless distance between the nozzle and the center of the middle magnet, $L / d$. Two regimes can be distinguished, separated by a critical Stuart number $\mathrm{N}_{c} \approx 0.01$. The Lorentz force is significant in this configuration, even for Stuart numbers far below one. This is due to the choice of the characteristic length and velocity scales in our definition of the Stuart number, as mentioned in Sec. III.

For $\mathrm{N}<\mathrm{N}_{c}$, inertia forces are dominant, the Strouhal number is constant and has the same value as in the case without Lorentz forces: $\mathrm{St}=0.012$ (Figure 6). For $\mathrm{N}>\mathrm{N}_{c}$, the Lorentz forces are dominant and the Strouhal number is proportional to the square root of the Stuart number, St $\propto \sqrt{\mathrm{N}}$. The constant of proportionality between St and $\sqrt{\mathrm{N}}$ is approximately 0.1 , as was the case for $v_{i n}=0$ in Sec. V.

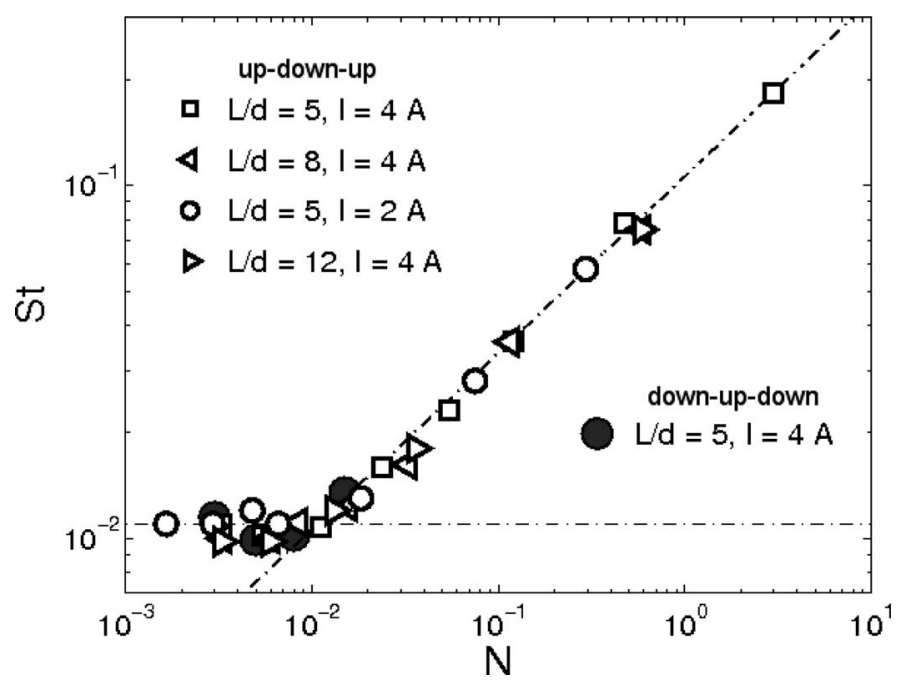

FIG. 9. Strouhal as a function of Stuart. The straight dash-dotted lines are the lines $\mathrm{St}=0.012$ and $\mathrm{St}=0.1 \sqrt{\mathrm{N}}$ and they intersect at the critical Stuart number, $\mathrm{N}_{c}=0.01$. 


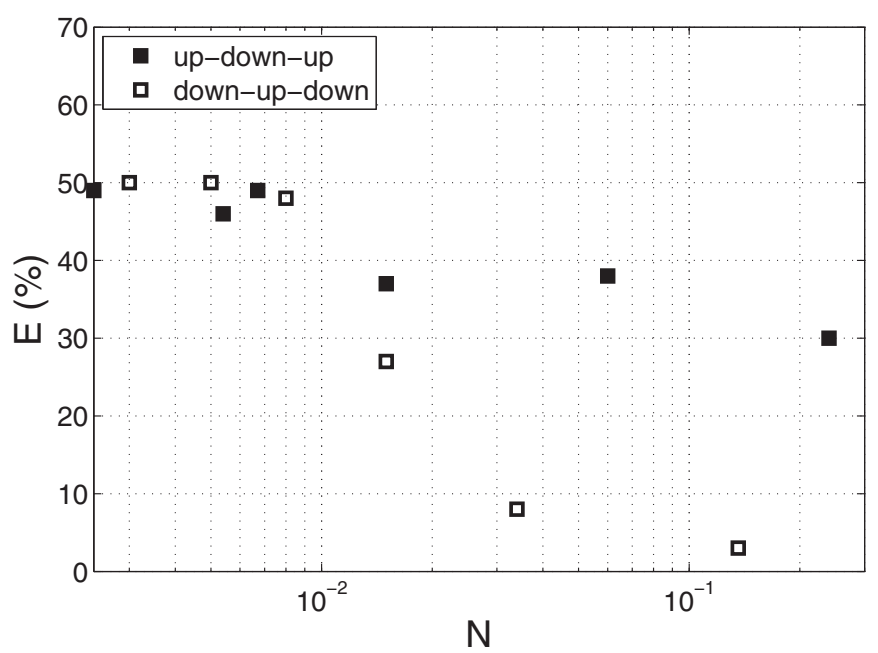

FIG. 10. Fraction of the kinetic energy contained in the first mode of the POD as a function of the Stuart number for the up-down-up and the down-up-down configuration.

\section{MIXED FORCING $(\mathrm{N}>0, \mathrm{Re}>0)$ : DOWN-UP-DOWN}

In addition to the results for up-down-up forcing, Figure 9 also shows St as a function of $\mathrm{N}$ for the down-up-down forcing configuration. For $\mathrm{N}<\mathrm{N}_{c} \approx 0.01$, St is again constant and equal to the value found for the up-down-up configuration and to the value found in the absence of Lorentz forces, indicating that inertia forces are dominant. For $\mathrm{N}>\mathrm{N}_{c}$, however, the oscillations are suppressed in the case of down-up-down forcing. Measurements have been performed for $\mathrm{N}>0.01$, all leading to full suppression of the oscillations and therefore not shown as data points in Figure 9.

Figure 10 shows the fraction of the energy contained in the first spatial mode of the POD as a function of the Stuart number. It is seen that for $\mathrm{N}>\mathrm{N}_{c} \approx 0.01$ the fraction of the kinetic energy contained in the first spatial mode sharply drops for the down-up-down configuration. This indicates the suppression of the oscillation and hence a stabilizing effect on the flow. To investigate the effect of the Lorentz force on the jet oscillations, mean velocity profiles, profiles of the turbulence kinetic energy, and results from the POD have been calculated. These will be discussed in Secs. VII A, VII B, and VII C, respectively. The analysis is done for flows with Reynolds numbers of $\operatorname{Re}=3150$ $(\mathrm{N}=0.05), \operatorname{Re}=4700(\mathrm{~N}=0.02)$, and $\mathrm{Re}=7100(\mathrm{~N}=0.007)$.

\section{A. Mean velocity field}

Figure 11 shows the mean velocity fields for the (oscillation suppressing) down-up-down forcing configuration (left) and for the configuration without Lorentz forces (right). The gray tone indicates the magnitude of the mean velocity $|u|=\sqrt{u_{x}^{2}+u_{y}^{2}}$, where a dark tone indicates a high velocity. The velocity fields are made dimensionless with the inlet velocity $v_{i n}$. For good visualization of the mean flow pattern, the values of the dimensionless velocity have been clipped at $|u| / v_{i n}=0.5$. As expected, the maximum velocity occurs in the near jet region in all cases. For $\operatorname{Re}=3150$ $(\mathrm{N}=0.05)$ and $\operatorname{Re}=4700(\mathrm{~N}=0.02)$ the flow induced by the Lorentz force results in the formation of recirculation zones in the top corners. These recirculation zones are absent for $\operatorname{Re}=7100$ $(\mathrm{N}=0.007)$.

The mean velocity fields in Figure 11 give a good indication of the global flow pattern in the cavity, but due to a lack of spatial resolution of the PIV measurements, accurate quantitative data cannot be determined from these measurements. Figure 12 shows quantitative results of the normalized velocity in the vertical direction, $u_{y}$, from PIV measurements with a smaller field of view zoomed in into the jet region. The field of view is $-d_{n}<y<-d_{n}-0.15 \mathrm{~m}$ and $-0.15<x / W<$ 0.15 . This results in a spatial resolution of $200 \mu \mathrm{m}$ per interrogation area and with $50 \%$ overlap 

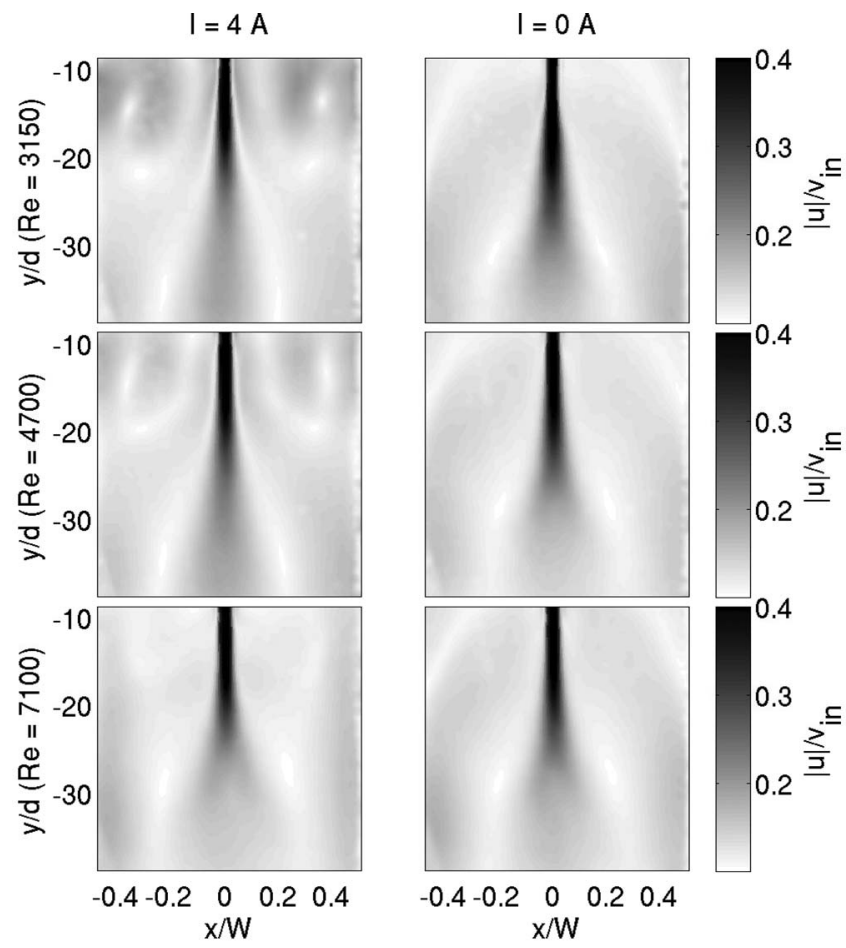

FIG. 11. Mean velocity magnitude made dimensionless with the inlet velocity for the down-up-down forcing configuration. From top to bottom, the values of the Stuart number in the left column are $\mathrm{N}=0.05, \mathrm{~N}=0.02$, and $\mathrm{N}=0.007$.

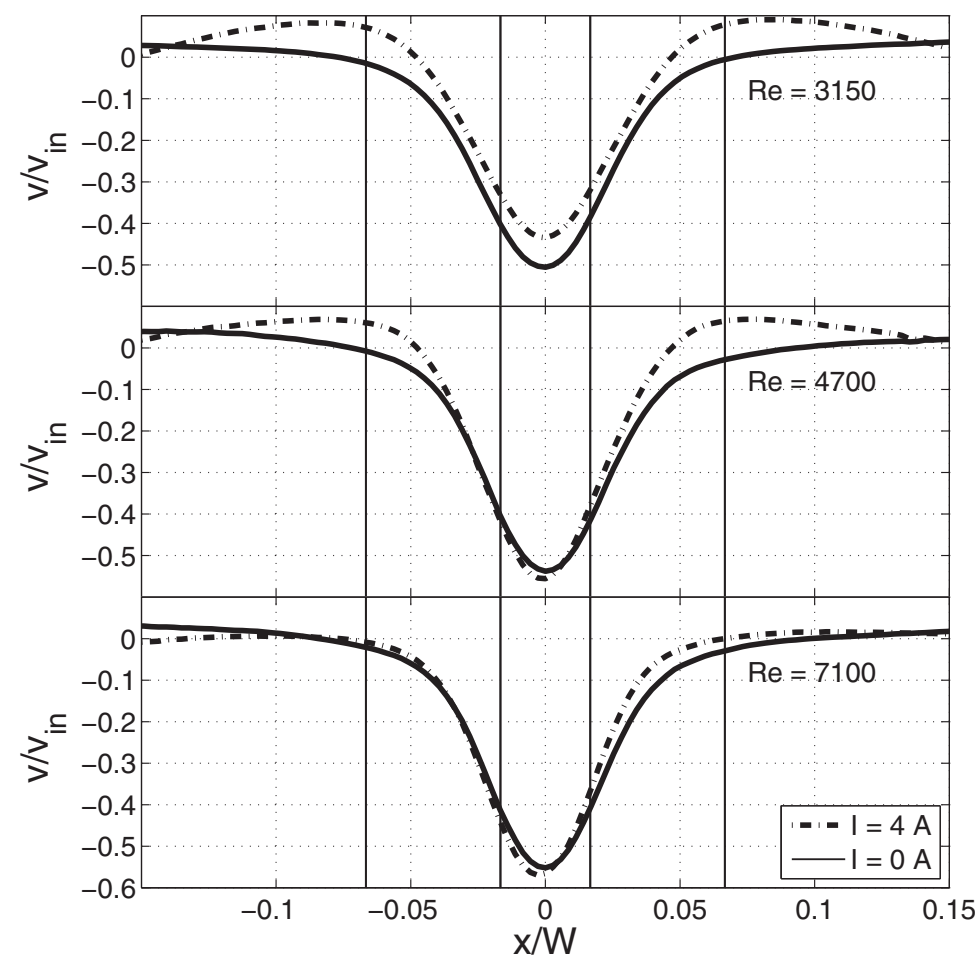

FIG. 12. Profiles of the $y$-component of the mean velocity $u_{y}$ at $y=-d_{n}-0.08 \mathrm{~m}$. The vertical lines at $|x / W|=0.017$ indicate the $x$-position of the inner nozzle walls and the vertical lines at $|x / W|=0.067$ indicate the $x$-position of the edges of the center magnet. 
between the interrogation areas this results in 1 velocity vector per $100 \mu \mathrm{m}$. The measurement time was $126 \mathrm{~s}$ at a sampling rate of $25 \mathrm{fps}$, resulting in 3155 image pairs. The velocity profiles shown here are taken at $y=-d_{n}-0.08 \mathrm{~m}$, i.e., 8 pipe diameters downstream from the nozzle exit. The $x$-position of the nozzle is indicated by the vertical lines at $|x / W|=0.017$. The edges of the center magnet are indicated by the vertical lines at $|x / W|=0.067$. Figure 12 shows that the peak velocity is reduced by $20 \%$ due to the Lorentz force when $\mathrm{Re}=3150(\mathrm{~N}=0.05)$. The velocities outside the jet region are affected in the cases with $\mathrm{Re}=3150(\mathrm{~N}=0.05)$ and $\mathrm{Re}=4700(\mathrm{~N}=0.02)$. For $|x / W|>0.05$ a reversal of the flow direction is seen, which is due to the upward directed Lorentz force. In the jet region this upward directed Lorentz force is overcome by the jet, but outside the jet region this results in an upward directed flow. When $\mathrm{Re}=7100(\mathrm{~N}=0.007)$ the flow reversal due to the Lorentz force has vanished, indicating that the flow in this case is dominated by the jet flow.

\section{B. Turbulence kinetic energy distribution}

The Reynolds decomposition of the horizontal velocity component is

$$
u_{x}=\overline{u_{x}}+u_{x}^{\prime},
$$

where $u_{x}$ is the instantaneous velocity component, $\overline{u_{x}}$ is the mean velocity, and $u_{x}^{\prime}$ is the velocity fluctuation associated with turbulence. With a similar decomposition for $u_{y}$, the two-dimensional turbulence kinetic energy is then calculated from

$$
k=1 / 2\left[\overline{u_{x}^{\prime 2}}+\overline{u_{y}^{\prime 2}}\right] .
$$

The two-dimensional representation of the turbulence kinetic energy is used since measurements are performed with a two-component PIV system.

Figure 13 shows the spatial distribution of the turbulence kinetic energy, made dimensionless with the square of the inlet velocity, calculated from the same measurement data used to calculate the mean velocity fields in Figure 11. The values of $k / v_{i n}^{2}$ have been clipped at $k / v_{i n}^{2}=0.025$ to provide a clearer picture of the global distribution of the turbulence kinetic energy. A peak in $k$ is located near the jet exit region in all three cases, when moving in the streamwise direction, the jet shear layer spreads while turbulence levels decrease. This is due to the entrainment of low velocity ambient fluid. Due to the limited spatial resolution of the PIV measurements in the jet region, the jet shear layers cannot be resolved in detail.

In absence of the Lorentz force $(I=0)$, two lobes are present in the turbulence kinetic energy distribution. These lobes are related to the extreme positions of the oscillating jet. When down-updown forcing is applied with an electrical current of $I=4.0 \mathrm{~A}$ it is seen that the strength of the lobes is reduced for $\operatorname{Re}=3150(\mathrm{~N}=0.05)$ and $\operatorname{Re}=4700(\mathrm{~N}=0.02)$. This indicates that the Lorentz force for the down-up-down configuration has a stabilizing effect on the large scale jet fluctuations. For $\mathrm{Re}=7100(\mathrm{~N}=0.007)$ the applied Lorentz forcing has no observable effect on the jet oscillations, indicating that the transition from electromagnetically dominated flow towards inertia dominated flow is between $\mathrm{N}=0.02$ and $\mathrm{N}=0.007$ in agreement with the earlier found critical Stuart number $\mathrm{N}_{c}=0.01$.

\section{Proper orthogonal decomposition}

A POD is performed on the PIV snapshots that were used to calculate the results shown in Figures 11 and 13. Values of the fraction of the turbulence kinetic energy contained in the first spatial POD mode, $\phi_{1}$, for Reynolds numbers of $3150(\mathrm{~N}=0.05), 4700(\mathrm{~N}=0.02)$, and 7100 $(\mathrm{N}=0.007)$ are listed in Table II. It is seen that the energy contained in the first POD mode is lowest for cases with Lorentz forcing and $\mathrm{Re}=3150(\mathrm{~N}=0.05)$. This indicates that at a relatively low inlet velocity, the large scale structures in the flow are stabilized considerably when the Lorentz force is applied. Figure 14 shows the energy per POD mode number $n$ as a fraction of the total kinetic energy for modes $1-30$. For $\operatorname{Re}=3150$, the distribution of the fraction of kinetic energy is affected significantly by the presence of electromagnetic forcing, leading to a more or less even distribution of the kinetic energy. For $\mathrm{Re}=7100$, the distributions of the fraction of the kinetic energy are equal 

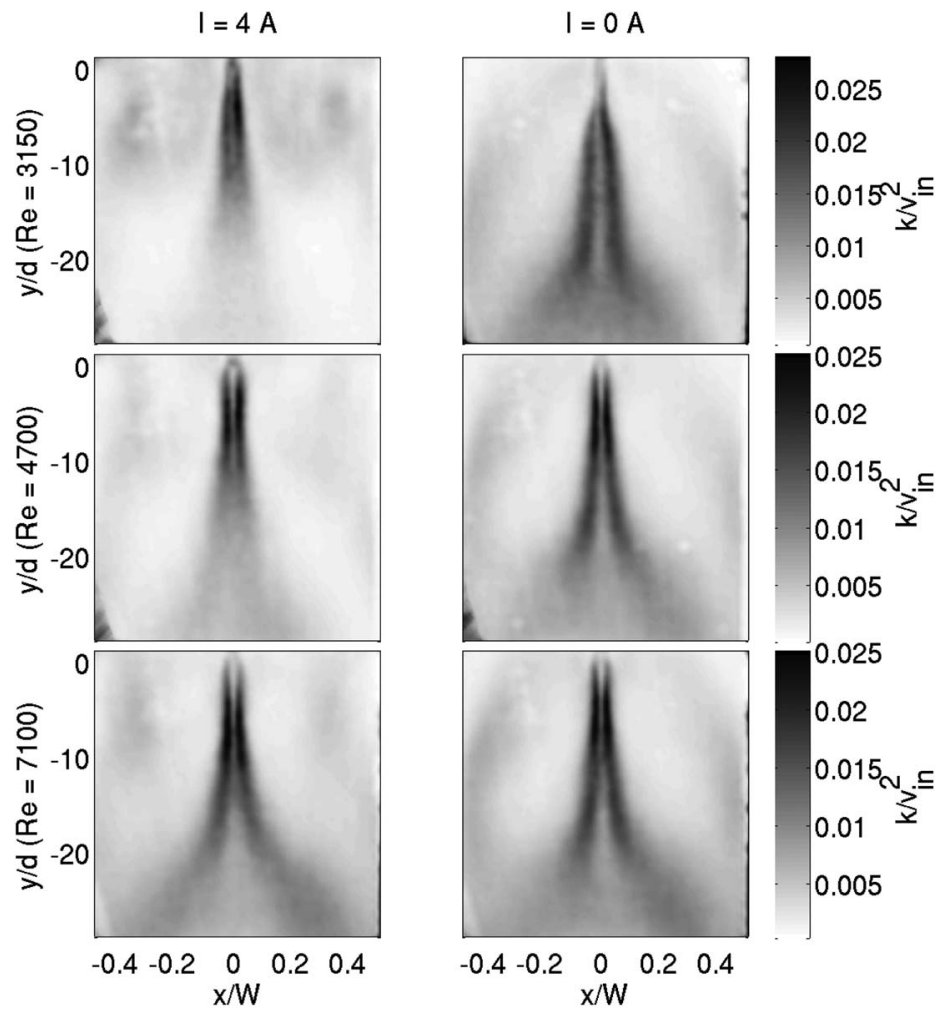

FIG. 13. Turbulence kinetic energy, determined from a set of 300 PIV velocity fields recorded at a frequency of 1 fps. From top to bottom, the values of the Stuart number in the left column are $\mathrm{N}=0.05, \mathrm{~N}=0.02$, and $\mathrm{N}=0.007$.

for the cases with and without electromagnetic forcing, leading to the conclusion that in this case the electromagnetic forcing does not affect the flow dynamics.

Figure 15 shows phase diagrams of the first and second temporal modes, $a_{1}(t)$ and $a_{2}(t)$, for the cases with and without Lorentz forcing for $\mathrm{Re}=3150(\mathrm{~N}=0.05), \mathrm{Re}=4700(\mathrm{~N}=0.02)$, and $\operatorname{Re}=7100(\mathrm{~N}=0.007)$. In flows with strong periodicities these temporal modes reduce to the first two Fourier modes, which have a phase difference of $90^{\circ}$. In the cases without Lorentz forcing, the coefficients show a closed curve of ellipsoidal shape. This indicates that $a_{1}(t)$ and $a_{2}(t)$ are periodic with the same frequency. The axes of the ellipsoid are aligned with the $x$ - and $y$-axis, indicating that the phase difference between $a_{1}(t)$ and $a_{2}(t)$ is $90^{\circ}$. This indicates that the large scale flow dynamics in these flows can be described using only two POD modes. When a Lorentz force is applied with $\mathrm{N}>0.02$, a deviation from the ellipsoidal shape is observed, indicating that the low dimensional description for the oscillating jet is not valid anymore. For $\mathrm{N}=0.05$, the coefficients $a_{1}(t)$ and $a_{2}(t)$ no longer form a closed curve, indicating that in this case more POD modes are necessary to describe the flow dynamics correctly.

TABLE II. Fraction of the turbulence kinetic energy contained in the first spatial mode of the POD, $\phi_{1}(\mathbf{x})$, for varying Reynolds numbers and with and without applied Lorentz forcing.

\begin{tabular}{lcc}
\hline \hline $\operatorname{Re}$ & $\mathrm{I}=0 \mathrm{~A}$ & $\mathrm{I}=4.0 \mathrm{~A}$ \\
\hline 3150 & $32 \%$ & $6 \%$ \\
4700 & $39 \%$ & $39 \%$ \\
7100 & $39 \%$ & $46 \%$ \\
\hline \hline
\end{tabular}



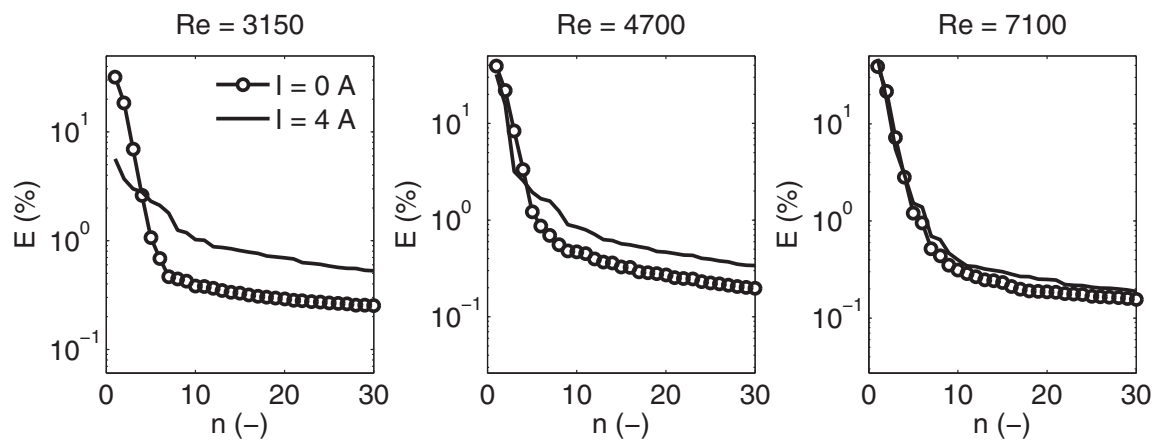

FIG. 14. Energy contained in POD modes 1-30 shown as a percentage of the total kinetic energy. From left to right the Stuart numbers are $\mathrm{N}=0.05, \mathrm{~N}=0.02$, and $\mathrm{N}=0.007$.

\section{PHYSICAL MECHANISM OF THE JET OSCILLATION}

Figure 16 schematically shows the main stages of the jet oscillation. Visual observation of the start up phase of the jet oscillation showed that the jet initially flows straight to the bottom of the cavity, inducing (nearly) symmetric recirculation zones on both sides of the jet (stage (a)). Once the high velocity liquid of the jet reaches the bottom of the cavity, the fluid preferentially moves either towards the left wall or the right wall. This is a random process, but in the description below it is assumed that the jet fluid moves towards the right. Then the fluid moves upward along the right wall thus forming a recirculation zone (stage (b)). The recirculation zone has a pressure minimum in its center that causes the jet fluid to bend towards the right, thus feeding the right recirculation zone. The jet bending continues to increase until the jet impinges on the side wall with the impingement point gradually moving upward. The impinging jet splits in an upward directed part and a downward directed part. The part of the jet fluid flowing towards the bottom of the cavity leads to the formation
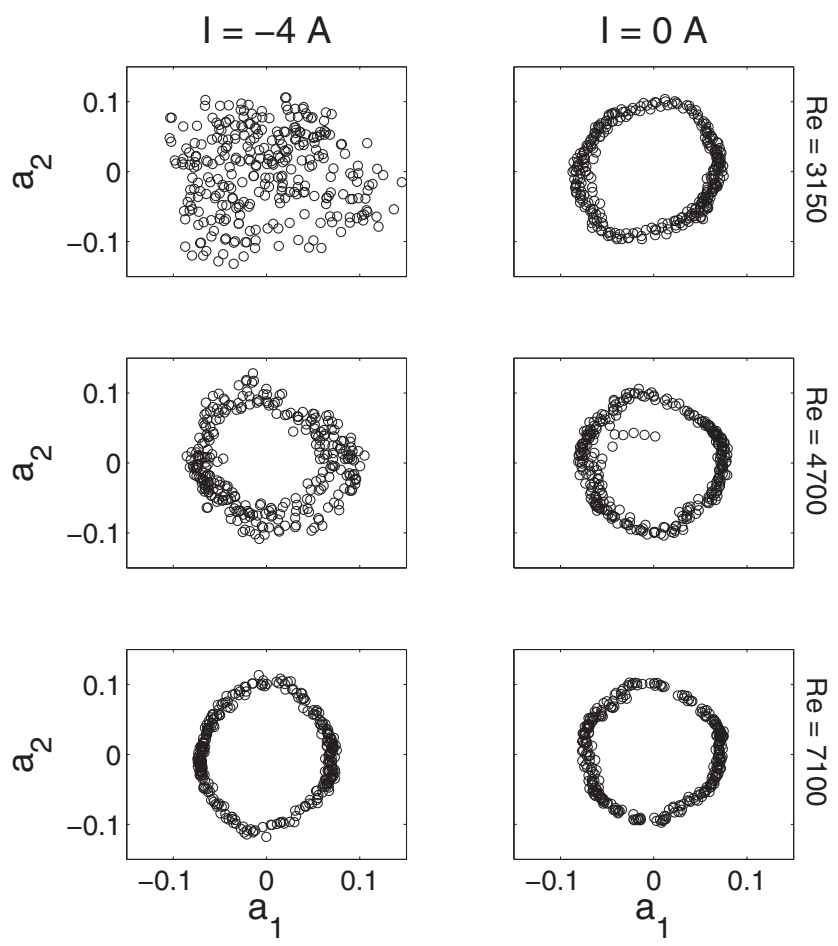

FIG. 15. Phase diagram of the first and second temporal POD coefficients $a_{1}(t)$ and $a_{2}(t)$. From top to bottom, the values of the Stuart number in the left column are $\mathrm{N}=0.05, \mathrm{~N}=0.02$, and $\mathrm{N}=0.007$. 


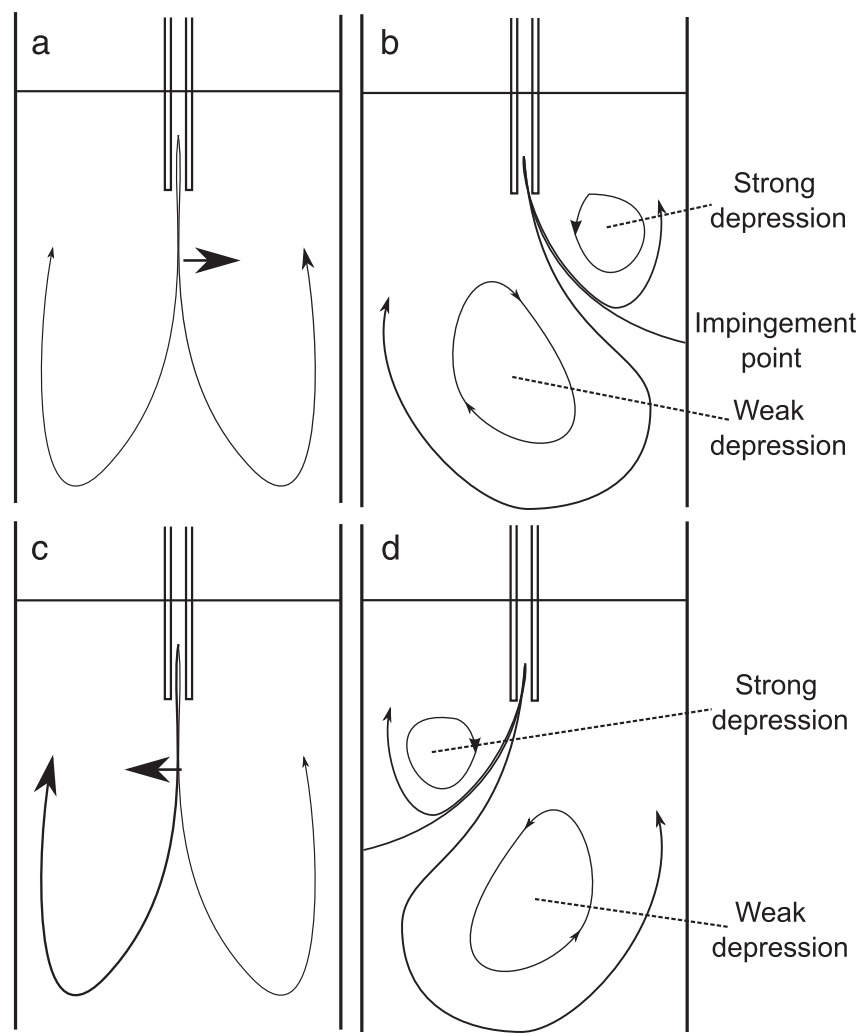

FIG. 16. Schematics of subsequent stages of the jet oscillation. (a) Initially the jet flows symmetrically towards the bottom of the cavity and then it is bended towards the right. (b) The jet has bended towards the recirculation zone on the right until it impinges on the right wall. The jet splits in an upward moving part and in a downward moving part. (c) The jet has moved to the neutral position $(\theta=0)$, moving towards the left. (d) The jet impinges on the left wall and splits in an upward moving part and in a downward moving part.

of a recirculation zone on the other side of the jet. The strength of this recirculation zone gradually increases thus increasing the pressure difference between the jet and the center of this recirculation zone. The jet is then drawn towards the left in stage (c) until it impinges on the side wall in stage (d) and from then on the process repeats itself.

To quantitatively investigate this oscillation mechanism, attention was focused on the temporal variation of the jet angle $\theta$ and the vertical fluid velocity $u_{y}$ on both sides of the jet, following the approach presented by Honeyands and Herbertson ${ }^{4}$ and in our previous publication. ${ }^{9}$ In Figure 17 (left) the jet angle $\theta$ is defined as the angle between (1) the vertical line $x=0$ and (2) the line through the position of the maximum jet velocity at $y=-0.3 \mathrm{~m}$ and the tip of the nozzle. The jet angle is assumed to be positive when the jet is directed to the right. The vertical fluid velocity on the left and on the right of the jet was monitored at $y=-0.175 \mathrm{~m}$ and $x= \pm 0.1 \mathrm{~m}$. When the liquid velocity is directed in the upward direction, the velocity is positive.

Figure 17 (right) shows both the jet angle and the vertical velocities for a jet Reynolds number of 3150 and a Lorentz force in the up-down-up configuration $(\mathrm{N}=0.05)$. Three characteristic events in the jet oscillation have been marked with numbers. At $t=35 \mathrm{~s}$ (1) the jet angle has a maximum, meaning that the jet is flowing towards the right wall. This is when the jet impinges on the side wall and splits in an upward and downward part (see Figure 16(b)). The velocity on the right side of the jet is decreasing, while the velocity on the left is increasing leading to the formation of a strong recirculation zone on the left. At $t=41 \mathrm{~s}(2)$ the velocity on the left side of the jet has a maximum and the velocity on the right has a minimum. The jet angle is still decreasing (Figure 16(c)). At $t=43 \mathrm{~s} \mathrm{(3)}$ the jet angle reaches its minimum, and at this point the velocity on the right is growing 

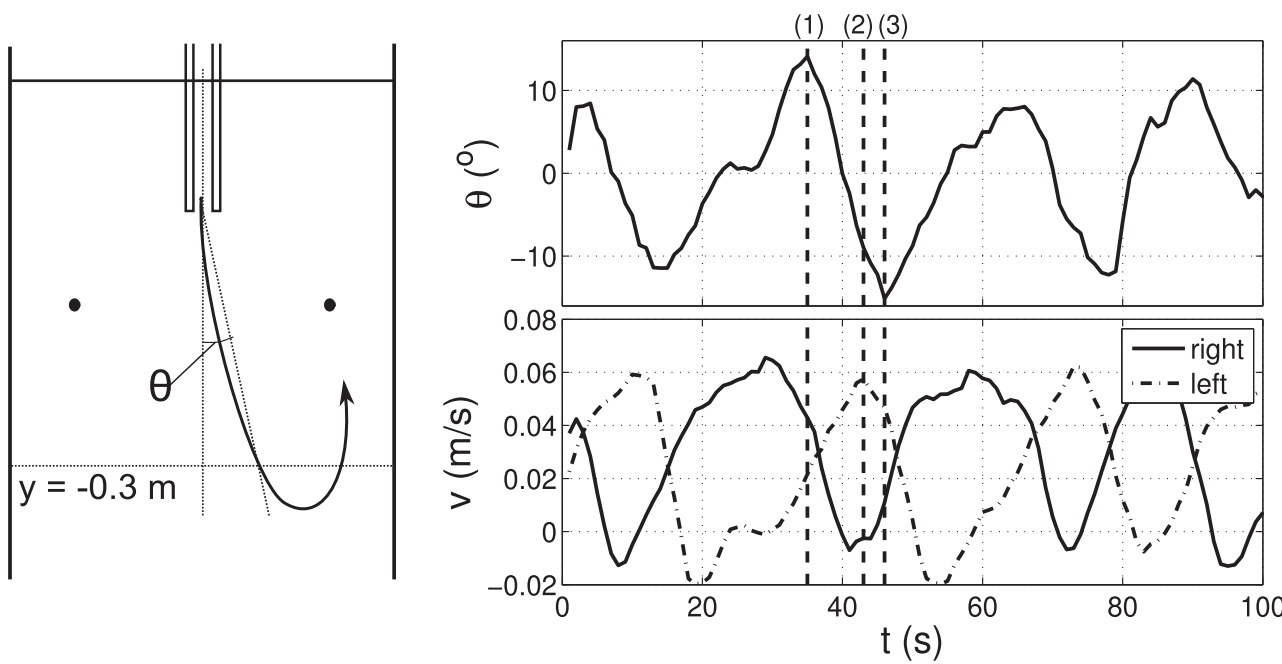

FIG. 17. Left: The jet angle $\theta$ is defined as the angle between the vertical line through $x=0$ and the line through the position of the maximum in the jet velocity at $y=-0.3 \mathrm{~m}$ and through the tip of the nozzle $\theta$. The dots on the left and right indicate where the vertical component of the velocity is monitored. Right, top: Jet angle development, a positive jet angle means that the jet is directed to the right. Right, bottom: Variation of the vertical velocity on the left and the right of the jet at $y=0.175$ $\mathrm{m}$ and $x= \pm 0.1 \mathrm{~m}$. A positive velocity means that the fluid moves in the upward direction. $\mathrm{Re}=3150$ and $\mathrm{N}=0.05$ with the Lorentz force in the up-down-up configuration.

and the velocity on the left is decreasing, leading to the formation of the strong recirculation cell on the right (Figure 16(d)). Now the same process repeats, leading to a stable jet oscillation.

When the Lorentz force is in the up-down-up configuration (as is the case for the results shown in Figure 17) the upward directed flow due to the Lorentz force on the right side of the jet adds to the growing velocity in the initial recirculation zone that forms on the right side of the jet. This leads to a faster growth of the velocity in the recirculation zone. When the jet angle has a maximum value and the velocity on the left starts to grow, this growth is enhanced by the presence of the upward directed flow due to the Lorentz force on the left side and the jet is drawn to the left much quicker than in the absence of the Lorentz force. The whole process of the formation of recirculation zones is faster leading to a higher oscillation frequency when the Lorentz force is applied in the up-down-up configuration as was already depicted in Figure 9.

When the Lorentz force is in the down-up-down configuration the flow due to the presence of the Lorentz force at the position of the left and right magnet is in the downward direction. This means that the formation of the recirculation zones is hampered by the Lorentz force. If the Lorentz force is of sufficient strength (in comparison to the inertial forces) it prevents the formation of the low pressure regions next to the jet. This means that the mechanism needed for the jet oscillation has disappeared, as was shown for the flow with $\mathrm{Re}=3150$ and $\mathrm{N}=0.05$.

\section{CONCLUSIONS}

The effect of Lorentz forcing on self-sustained oscillations of a liquid jet flowing into a liquid filled thin cavity has been studied. The Lorentz force was imposed on the fluid by applying a magnetic field in a north-south-north configuration in conjunction with an electric field perpendicular to the magnetic field. The Lorentz force could be either in the up-down-up configuration or in the downup-down configuration, by reversing the electrical current.

A critical Stuart number of $\mathrm{N}_{c} \approx 0.01$ was found. For $\mathrm{N}<\mathrm{N}_{c}$, the jet oscillates with a constant Strouhal number, $\mathrm{St}=0.012$, for $1.5 \times 10^{3}<\mathrm{Re}<3.0 \times 10^{4}$. For $\mathrm{N}>\mathrm{N}_{c}$ in the (oscillation enhancing) up-down-up configuration, St grows with $\mathrm{N}$ as $\mathrm{St} \approx 0.1 \sqrt{\mathrm{N}}$. In contrast, for $\mathrm{N}>\mathrm{N}_{c}$ in the (oscillation suppressing) down-up-down configuration, oscillations are suppressed. 


\section{ACKNOWLEDGMENTS}

This work was supported by the Dutch Technology Foundation STW, Tata Steel, and ABB.

${ }^{1}$ D. Rockwell and E. Naudascher, "Self-sustained oscillations of impinging free shear layers," Annu. Rev. Fluid Mech. 11, 67-94 (1979).

${ }^{2}$ D. Rockwell, "Oscillations of impinging shear layers," AIAA J. 21, 645-664 (1983).

${ }^{3}$ N. A. Molloy and P. L. Taylor, "Oscillatory motion of a jet into a blind cavity," Nature (London) 224, 1192-1194 (1969).

${ }^{4}$ T. A. Honeyands and J. Herbertson, "Flow dynamics in thin slab casting moulds," Steel Research 66, 287-293 (1995).

${ }^{5}$ A. Maurel, P. Ern, B. J. A. Zielinska, and J. E. Wesfreid, "Experimental study of self-sustained oscillations in a confined jet," Phys. Rev. E 54, 3643-3651 (1996).

${ }^{6}$ N. J. Lawson and M. R. Davidson, "Self-sustained oscillation of a submerged jet in a thin rectangular cavity," J. Fluid Struct. 15, 59-81 (2001).

${ }^{7}$ T. Kolšek, N. Jelić, and J. Duhovnik, "Numerical study of flow asymmetry and self-sustained jet oscillations in geometrically symmetric cavities," Appl. Math. Model. 31, 2355-2373 (2007).

${ }^{8}$ A. Mataoui and R. Schiestel, "Unsteady phenomena of an oscillating turbulent jet flow inside a cavity: Effect of aspect ratio," J. Fluid Struct. 25, 60-79 (2009).

${ }^{9}$ R. Kalter, M. J. Tummers, S. Kenjereš, B. W. Righolt, and C. R. Kleijn, "Oscillations of the fluid flow and the free surface in a cavity with a submerged bifurcated nozzle," Int. J. Heat Fluid Flow 44, 365-374 (2013).

${ }^{10}$ H. Yokoyama, Y. Tsukamoto, C. Kato, and A. Iida, "Self-sustained oscillations with acoustic feedback in flows over a backward-facing step with a small upstream step," Phys. Fluids 19, 106104 (2007).

${ }^{11}$ D. Wee, T. Yi, A. Annaswamy, and A. F. Ghoniem, "Self-sustained oscillations and vortex shedding in backward-facing step flows: Simulation and linear instability analysis," Phys. Fluids 16, 3361-3373 (2004).

${ }^{12}$ C. K. W. Tam and P. J. W. Block, "On the tones and pressure oscillations induced by flow over rectangular cavities," J. Fluid Mech. 89, 373-399 (1978).

${ }^{13}$ D. Rockwell and E. Naudascher, "Review - Self-sustaining oscillations of flow past cavities," ASME T. J. Fluids Eng. 100, 152-165 (1978).

${ }^{14}$ C. W. Rowley, T. Colonius, and A. J. Basu, "On self-sustained oscillations in two-dimensional compressible flow over rectangular cavities," J. Fluid Mech. 455, 315-346 (2002).

${ }^{15}$ C. W. Rowley and D. R. Williams, "Dynamics and control of high-Reynolds-number flow over open cavities," Annu. Rev. Fluid Mech. 38, 251-276 (2006).

${ }^{16} \mathrm{C}$. Henoch and J. Stace, "Experimental investigation of a salt water turbulent boundary layer modified by an applied streamwise magnetohydrodynamic body force," Phys. Fluids 7, 1371-1383 (1995).

${ }^{17}$ C. H. Crawford and G. E. Karniadakis, "Reynolds stress analysis of EMHD-controlled wall turbulence. Part I. Streamwise forcing," Phys. Fluids 9, 788-806 (1997).

${ }^{18}$ S. Kenjereš, "Electromagnetic enhancement of turbulent heat transfer," Phys. Rev. E 78, 066309 (2008).

${ }^{19}$ S. Kenjereš, J. Verdoold, M. J. Tummers, K. Hanjalić, and C. R. Kleijn, "Numerical and experimental study of electromagnetically driven vortical flows," Int. J. Heat Fluid Flow 30, 494-504 (2009).

${ }^{20}$ S. Kenjereš, "Electromagnetically driven dwarf tornados in turbulent convection," Phys. Fluids 23, 015103 (2011).

${ }^{21}$ L. Rossi, D. Doorly, and D. Kustrin, "Lamination and mixing in three fundamental flow sequences driven by electromagnetic body forces," Phys. Rev. E 86, 026313 (2012).

${ }^{22}$ L. Rossi, D. Doorly, and D. Kustrin, "Lamination and mixing in laminar flows driven by lorentz body forces," Europhys. Lett. 97, 14006 (2012).

${ }^{23}$ T. W. Berger, J. Kim, C. Lee, and J. Lim, "Turbulent boundary layer control utilizing the lorentz force," Phys. Fluids 12, 631-649 (2000).

${ }^{24}$ E. Spong, J. A. Reizes, and E. Leonardi, “Efficiency improvements of electromagnetic flow control,” Int. J. Heat Fluid Flow 26, 635-655 (2005).

${ }^{25}$ S. J. Kim and C. M. Lee, "Investigation of the flow around a circular cylinder under the influence of an electromagnetic force," Exp. Fluids 28, 252-260 (2000).

${ }^{26}$ H. Zhang, B. Fan, and Z. Chen, "Computations of optimal cylinder flow control in weakly conductive fluids," Comput. Fluids 39, 1261-1266 (2010).

${ }^{27}$ T. Weier, G. Gerbeth, G. Mutschke, O. Lielausis, and G. Lammers, "Control of flow separation using electromagnetic forces," Flow Turbul. Combust. 71, 5-17 (2003).

${ }^{28}$ T. Weier, C. Cierpka, and G. Gerbeth, "Coherent structure eduction from piv data of an electromagnetically forced separated flow," J. Fluid Struct. 24, 1339-1348 (2008).

${ }^{29}$ C. Cierpka, T. Weier, and G. Gerbeth, "Evolution of vortex structures in an electromagnetically excited separated flow," Exp. Fluids 45, 943-953 (2008).

${ }^{30}$ A. V. Wolf, M. G. Brown, and P. G. Prentiss, CRC Handbook of Chemistry and Physics (CRC Press, USA, 1986).

${ }^{31}$ L. Sirovich, "Turbulence and the dynamics of coherent structures .1. Coherent structures," Q. Appl. Math. 45, 561-571 (1987).

${ }^{32}$ P. Holmes, J. L. Lumley, and G. Berkooz, Turbulence, Coherent Structures, Dynamical Systems and Symmetry (Cambridge University Press, UK, 1996).

${ }^{33}$ R. J. Adrian and J. Westerweel, Particle Image Velocimetry (Cambridge University Press, UK, 2011).

${ }^{34}$ O. Semeraro, G. Bellani, and F. Lundell, "Analysis of time-resolved PIV measurements of a confined turbulent jet using POD and koopman modes,” Exp. Fluids 53, 1203-1220 (2012). 
${ }^{35} \mathrm{O}$. Uzol and C. Camci, "Experimental and computational visualization and frequency measurements of the jet oscillation inside a fluidic oscillator," J. Visual. 5, 263-272 (2002).

${ }^{36}$ A. Figueroa, F. Demiaux, S. Cuevas, and E. Ramos, "Electromagnetically driven vortices in a dipolar magnetic field in a shallow layer," J. Fluid Mech. 641, 245-261 (2009).

${ }^{37}$ M. Duran-Matute, R. R. Trieling, and G. J. F. van Heijst, "Scaling and asymmetry in an electromagnetically forced dipolar flow structure," Phys. Rev. E 83, 016306 (2011). 